\title{
Comparison of the perioperative outcomes in antero-superior mediastinal tumor resection performed by transcervical resection and video-assisted thoracoscopic surgery
}

\author{
Xin-Sheng Zhu ${ }^{1 \#}$, Nan Song ${ }^{1 \#}$, Nai-Cheng Song ${ }^{1}$, Alan Dart Loon Sihoe ${ }^{2}$, Wen-Xin He ${ }^{1}$, Ming Liu ${ }^{1}$, \\ Ge-Ning Jiang ${ }^{1}$, Peng Zhang ${ }^{1}$ \\ ${ }^{1}$ Department of Thoracic Surgery, Shanghai Pulmonary Hospital, Tongji University, Shanghai 200433, China; ${ }^{2}$ Department of Surgery, The Li Ka \\ Shing Faculty of Medicine, The University of Hong Kong, Hong Kong, China \\ Contributions: (I) Conception and design: GN Jiang, P Zhang; (II) Administrative support: P Zhang; (III) Provision of study materials or patients: \\ N Song; (IV) Collection and assembly of data: WX He, M Liu; (V) Data analysis and interpretation: N Song, XS Zhu, NC Song; (VI) Manuscript \\ writing: All authors; (VII) Final approval of manuscript: All authors. \\ \#These authors contributed equally to this work. \\ Correspondence to: Ge-Ning Jiang; Peng Zhang. Department of Thoracic Surgery, Shanghai Pulmonary Hospital, Tongji University, No. 507 \\ Zhengmin Road, Yangpu District, Shanghai 200433, China. Email: jgnwp@aliyun.com; zhangpeng0726@yeah.net.
}

Background: It remains controversial that whether transcervical resection (TC) was associated with better outcomes than video-assisted thoracoscopic surgery (VATS) in the treatment of antero-superior mediastinal tumors. We aimed to compare the safety and reliability between TC and VATS.

Methods: Between 2010 and 2012, 80 consecutive patients underwent antero-superior mediastinal tumor resection via TC $(n=31)$ or VATS $(n=49)$. Perioperative outcomes were compared. A propensity score-matched analysis was performed to control the potential confounders.

Results: A total of 41 men and 39 women with median age of 52.5 years were enrolled. No patient died during the perioperative course. After propensity matching, TC group was associated with less intraoperative blood loss $(35.1 \pm 18.7$ vs. $93.7 \pm 136.1 \mathrm{~mL}, \mathrm{P}=0.034)$, less postoperative drainage $(65.6 \pm 76.8$ vs. $335.0 \pm 154.9 \mathrm{~mL}, \mathrm{P}<0.001)$, shorter length of postoperative hospital stay $(3.2 \pm 1.2$ vs. $4.1 \pm 1.3$ days, $\mathrm{P}=0.003)$ and less hospitalization expense $(22,252.3 \pm 4,761.7$ vs. $26,514.2 \pm 4,052.8 \mathrm{CNY}, \mathrm{P}=0.002)$ compared to VATS group. One patient with VATS was converted to open surgery due to intraoperative vessels damage. The postoperative complication was null in TC group while it was $6.1 \%(\mathrm{n}=3)$ in VATS group $(\mathrm{P}=0.279)$, including 1 case of prolonged chest tube drainage and 2 cases of recurrent laryngeal nerve injury.

Conclusions: TC for antero-superior mediastinal tumors is a safe procedure with better perioperative outcomes compared to VATS.

Keywords: Transcervical resection (TC); video-assisted thoracoscopic surgery (VATS); mediastinal tumors; perioperative outcomes

Submitted Aug 07, 2018. Accepted for publication Nov 16, 2018.

doi: $10.21037 /$ jtd.2018.11.114

View this article at: http://dx.doi.org/10.21037/jtd.2018.11.114

\section{Introduction}

Surgical resection is the main therapeutic method for mediastinal tumors $(1,2)$. The common approaches for mediastinal tumor include sternotomy, thoracotomy, robotassisted thoracoscopic surgery, video-assisted thoracoscopic surgery (VATS) and transcervical resection (TC) (3), and the selection of approach is based on the location of tumor in the mediastinum $(4,5)$.

Median sternotomy is recognized as the gold standard for anterior mediastinal tumor over the last decades (6). 


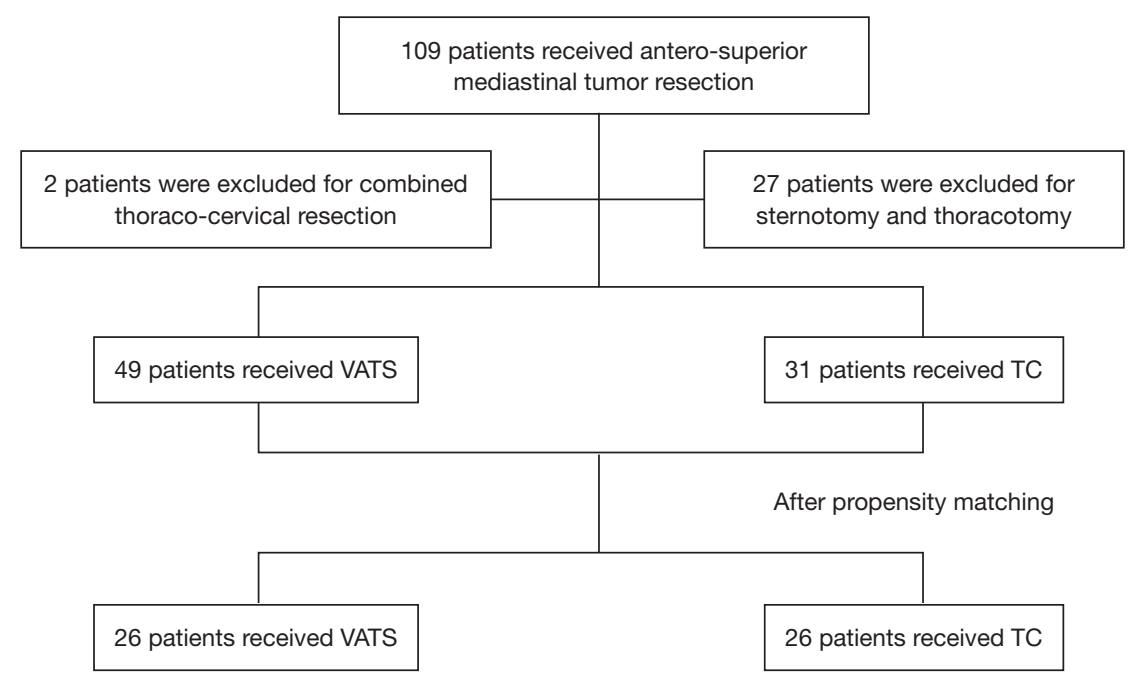

Figure 1 Flow diagram of patient recruitment. TC, transcervical resection; VATS, video-assisted thoracoscopic surgery.

However, sternotomy and thoracotomy are considered as more invasive approaches and associated with increased surgical trauma and postoperative complications, contributing to a prolonged hospitalization and long recovery time $(7,8)$. Compared to traditional surgical management, both VATS and TC offer excellent operative visualization with a decreased postoperative morbidity rate due to their minimal invasiveness. In addition, patients' subjective cosmetic satisfaction is significantly higher following VATS and TC approaches (9).

VATS was introduced into the thoracic field and applied to mediastinal tumor resection in the 1990s $(10,11)$. VATS has been demonstrated to be a safe procedure in the treatment of mediastinal tumors when the major vessels and organs are not invaded (12-14). As another minimally invasive approach for mediastinal tumors, TC was firstly reported in the 1910s (15). Since then, TC had been reported in several reports (16-19). We reviewed the cases with mediastinal tumor in our hospital and compared the perioperative outcomes between TC and VATS, to demonstrate the better approach for antero-superior mediastinal tumors.

\section{Methods}

\section{Study population and data collection}

This study was approved by the Institutional Review Board of Tongji University.

The same surgical indications of VATS and TC were applied to patients with symptomatic mediastinal masses or masses highly suspected of thymoma. Patients with anterosuperior mediastinal tumors were included. Patients were excluded from the study when they had a history of thoracic or cervical surgery, mass diameter was more than $5 \mathrm{~cm}$ on computed tomography scan, and mass directly invaded the surrounding tissue. All the patients were operated by the same group of operators.

From May 2010 to May 2012, 109 consecutive patients who underwent antero-superior mediastinal tumor resection with curative intent at Shanghai Pulmonary Hospital of Tongji University were included in this study. All the patients were approved by the Institutional Review Board of Tongii University. Of these, 27 patients with tumor invasion to major vessels received sternotomy or thoracotomy, 2 patients received combined thoraco-cervical resection, and these patients were excluded. Among the remaining 80 patients, 49 patients received VATS and 31 patients received TC (Figure 1).

None of these patients had comorbidity and received adjuvant treatment. All the masses were evaluated by the chest computed tomography scan (Figure 2). Whether patients should receive surgical treatment was determined by the multidisciplinary team. Written informed consent was obtained from all patients before operation. Complete resection (R0) was achieved in all cases.

The perioperative analgesic protocol for patients were similar between two groups. A patient-controlled analgesic system with intravenous morphine was provided on perioperative day one. Oral oxycodone (5 mg) 

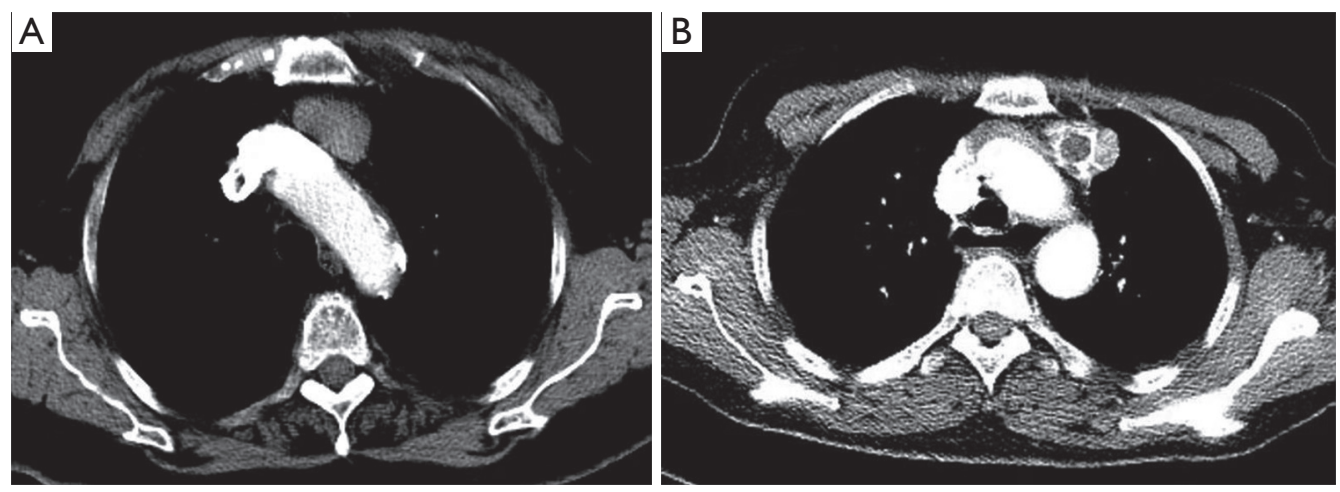

Figure 2 The computed tomography images of mediastinal masses. (A) A 68-year-old female in TC group presented an antero-superior mediastinal mass, with its largest diameter measurement of $3.1 \mathrm{~cm}$. The postoperative pathology confirmed to be type B3 thymoma. (B) A 59-year-old female in VATS group presented an antero-superior mediastinal mass, with its largest diameter measurement of $3.3 \mathrm{~cm}$. The postoperative pathology confirmed to be type AB thymoma. TC, transcervical resection; VATS, video-assisted thoracoscopic surgery.

were administrated three times a day and stopped on postoperative day four conventionally. The intensity of postoperative pain was evaluated using a visual analog scale score ranging from 0 (no pain) to 10 (maximal pain). This scale was taken from the NRS numerical rating scale (20). Pain score was recorded in our hospital information system in the daily progress note.

Clinical information was collected from the hospital information system and telephone interviews. Age, sex, operation time, intraoperative blood loss, incision length, postoperative drainage, pain score, postoperative hospital stay, hospitalization costs and postoperative complications were compared.

Perioperative mortality was defined as death occurring during the same hospitalization or within 30 days after operation. Prolonged intubation was defined as intubation time longer than 48 hours after surgery. Prolonged chest tube drainage was defined as drainage time longer than 7 days. Criteria for chest tube removal included no air leakage, lung re-expansion and the postoperative drainage below $200 \mathrm{~mL} /$ day. Criteria for patient discharge were that routine blood test and blood biochemistry indices were in normal range. Postoperative evaluation was performed by the chest computed tomography scan at 6 and 12 months after surgery, and annually thereafter.

\section{Surgical procedure}

Our operative technique of TC was as follows: patients were positioned in a supine position on the operating table with an inflatable bag placed beneath the shoulder after general anesthesia and intubation. A $2-3 \mathrm{~cm}$ incision was made along the natural skin lines $2 \mathrm{~cm}$ above the sternal notch. The strap muscles were divided on the midline and the sternum was lifted with a sternal retractor. Retractors improved by our department were placed at the edge of the incision against the upward pull of the sternal support. Illumination was provided with a headlight on the surgeons (Figure 3). After complete resection of the tumors and surrounding tissues, the drainage tubes were inserted through the incision (Figure 4).

Our operative technique of VATS was as follows: Patients were positioned in a $30^{\circ}$ semi-supine position on the operating table with a roll placed beneath the shoulder and the ipsilateral arm held abducted over a padded L-screen to expose the axilla. Lung isolation was obtained with a double lumen endotracheal tube ventilation. The side we entered into the chest depended on the location of tumors. All the patients received single port VATS. A $3 \mathrm{~cm}$ incision was made in the 3 th or 4 th intercostal space at the anterior axillary line. After resecting tumors and the surrounding tissue completely, one chest drainage tube was placed at the end of the incision wound.

\section{Statistical analysis}

Continuous variables were expressed as mean value and standard deviation (SD) and were compared by independent-sample $t$-test or Mann-Whitney $\mathrm{U}$ test. Categorical variables were expressed as frequencies with corresponding percentages and were compared by Chi-square test or Fisher's exact tests. For a more reliable comparison, we performed propensity score- 

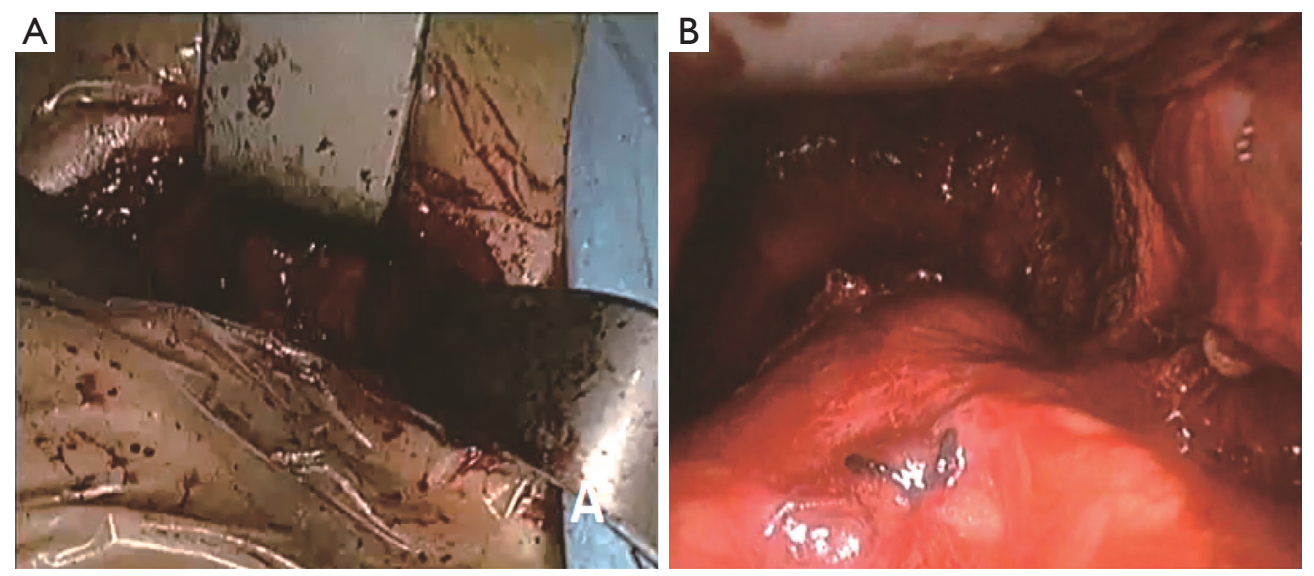

Figure 3 Our previous operative technique of TC. (A) The sternum was lifted with a sternal retractor and improved retractors were placed at the edge of the incision. (B) The exposure of the antero-superior mediastinum. TC, transcervical resection.
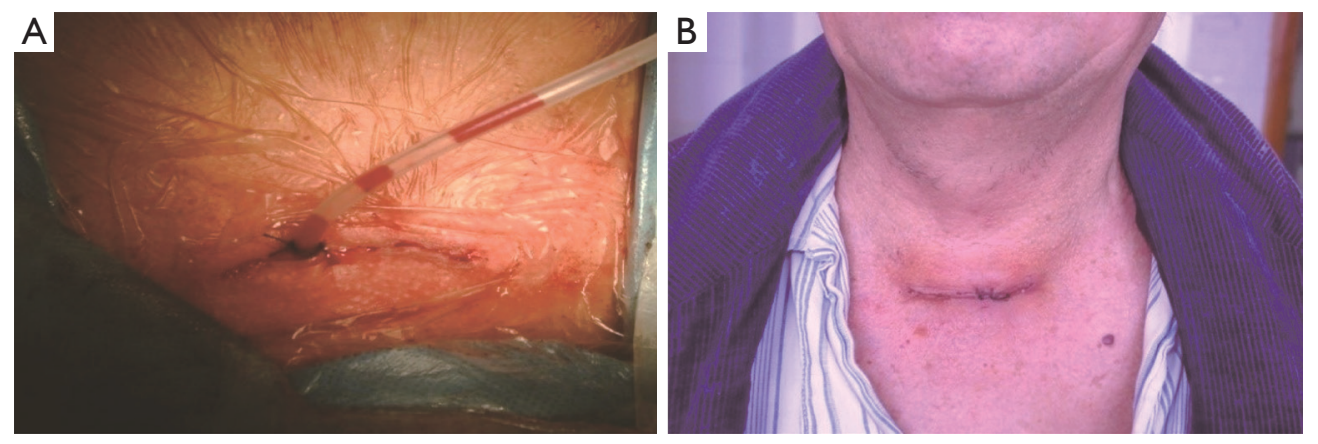

Figure 4 A relatively short incision by TC resection. (A) Drainage tube was place at the incision. (B) The incision healed nicely after operation. TC, transcervical resection.

based matching (TC:VATS $=1: 1$ ) based on baseline characteristics (age, gender, tumor diameter and tumor characteristic: solid or cyst). Two-tailed $\mathrm{P}$ values of $<0.05$ were considered statistically significant. All calculations were analyzed with the Statistical Package for Social Sciences (SPSS) Statistics, version 22 (IBM Corp Released 2013. Armonk, NY, USA).

\section{Results}

The characteristics of the patients in two groups were described in Table 1. A total of 41 men and 39 women (median age 52.5 years; range, 14-85 years) were enrolled. No statistically significant difference was found in gender, age and tumor characteristic between TC and VATS groups. The postoperative outcomes of the patients in two groups were described in Table 2 . No statistically significant difference was found in operative time $(111.3 \pm 55.7 v s$.
$124.3 \pm 51.6 \mathrm{~min}, \mathrm{P}=0.291)$ between the two groups. The incision length $(2.9 \pm 0.9$ vs. $5.1 \pm 1.0 \mathrm{~cm})$, intraoperative blood loss $(36.2 \pm 21.4 v s .98 .3 \pm 125.8 \mathrm{~mL})$, postoperative drainage $(82.1 \pm 83.6 v s .342 .4 \pm 185.3 \mathrm{~mL})$, pain score on postoperative day one $(2.6 \pm 1.4 v s .3 .4 \pm 1.7)$, pain score on discharge day $(1.4 \pm 0.7$ vs. $2.3 \pm 0.8)$, postoperative hospital stay $(3.5 \pm 1.3 v s$. $4.2 \pm 1.6$ days $)$ and hospitalization costs $(22,764.3 \pm 4,943.2$ vs. 26,958.4 $\pm 3,425.0 \mathrm{CNY}$ ) were significantly lower in TC group than VATS group (all $\mathrm{P}<0.05$ ). The postoperative pathological results in two groups were described in Table 3 , all the tumors included were benign tumors or were in very early stage thymoma, and there was no significant difference in the proportion of tumors in two groups $(\mathrm{P}=0.528)$. The median follow-up time of the two groups was 62.5 months, with 61.5 months in TC group and 64.0 months in VATS group, respectively. During the follow-up, no patients developed recurrent tumors or dead of tumor-related cause.

Although no significant difference was noted in 
Table 1 Clinical characteristics of patients before and after PSM

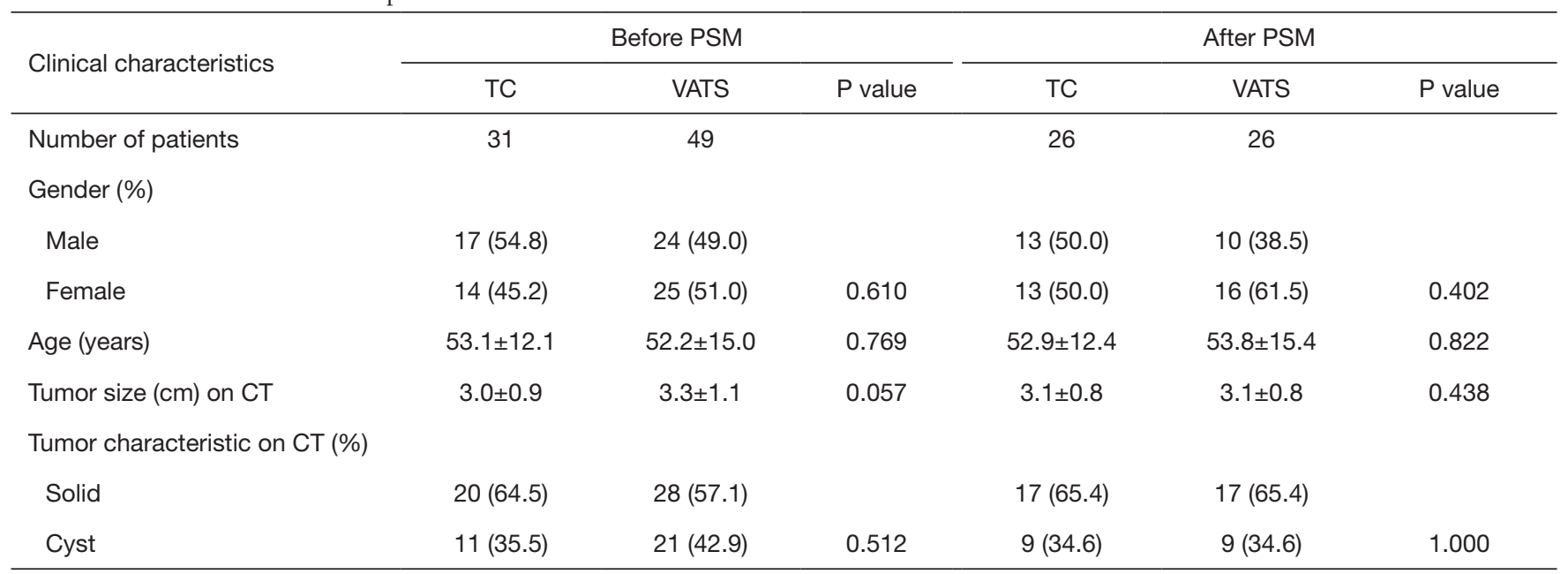

PSM, propensity score match; TC, transcervical resection; VATS, video-assisted thoracoscopic surgery.

Table 2 Perioperative outcomes of patients before and after PSM

\begin{tabular}{|c|c|c|c|c|c|c|}
\hline Perioperative outcomes & \multicolumn{3}{|c|}{ Before PSM } & \multicolumn{3}{|c|}{ After PSM } \\
\hline Number of patients & 31 & 49 & & 26 & 26 & \\
\hline Operative time (min) & $111.3 \pm 55.7$ & $124.3 \pm 51.6$ & 0.291 & $112.1 \pm 58.3$ & $116.5 \pm 40.1$ & 0.757 \\
\hline Incision lengths $(\mathrm{cm})$ & $2.9 \pm 0.9$ & $5.1 \pm 1.0$ & $<0.001$ & $3.0 \pm 0.9$ & $5.1 \pm 1.1$ & $<0.001$ \\
\hline Postoperative drainage (mL) & $82.1 \pm 83.6$ & $342.4 \pm 185.3$ & $<0.001$ & $65.6 \pm 76.8$ & $335.0 \pm 154.9$ & $<0.001$ \\
\hline Pain score on postoperative day 1 & $2.6 \pm 1.4$ & $3.4 \pm 1.7$ & 0.032 & $2.3 \pm 1.0$ & $3.0 \pm 1.5$ & 0.046 \\
\hline Pain score on discharge day & $1.4 \pm 0.7$ & $2.3 \pm 0.8$ & 0.046 & $1.4 \pm 0.6$ & $2.1 \pm 0.8$ & 0.345 \\
\hline Postoperative hospital stays (d) & $3.5 \pm 1.3$ & $4.2 \pm 1.6$ & 0.037 & $3.2 \pm 1.2$ & $4.1 \pm 1.3$ & 0.003 \\
\hline
\end{tabular}

PSM, propensity score match; TC, transcervical resection; VATS, video-assisted thoracoscopic surgery.

Table 3 Postoperative pathological diagnosis

\begin{tabular}{lcc}
\hline Pathology & TC group (\%) & VATS group (\%) \\
\hline Number of patients & 31 & 49 \\
Thymoma & $12(38.7)$ & $18(36.7)$ \\
Thymus benign tumor & $12(38.7)$ & $20(40.8)$ \\
Bronchial cyst & $5(16.1)$ & $6(12.2)$ \\
Ectopic thyroid & $2(6.5)$ & $1(2.0)$ \\
Teratoma & 0 & $2(4.0)$ \\
Pericardial cyst & 0 & $1(2.0)$ \\
Hemangioendothelioma & 0 & $1(2.0)$ \\
\hline
\end{tabular}

TC, transcervical resection; VATS, video-assisted thoracoscopic surgery. 

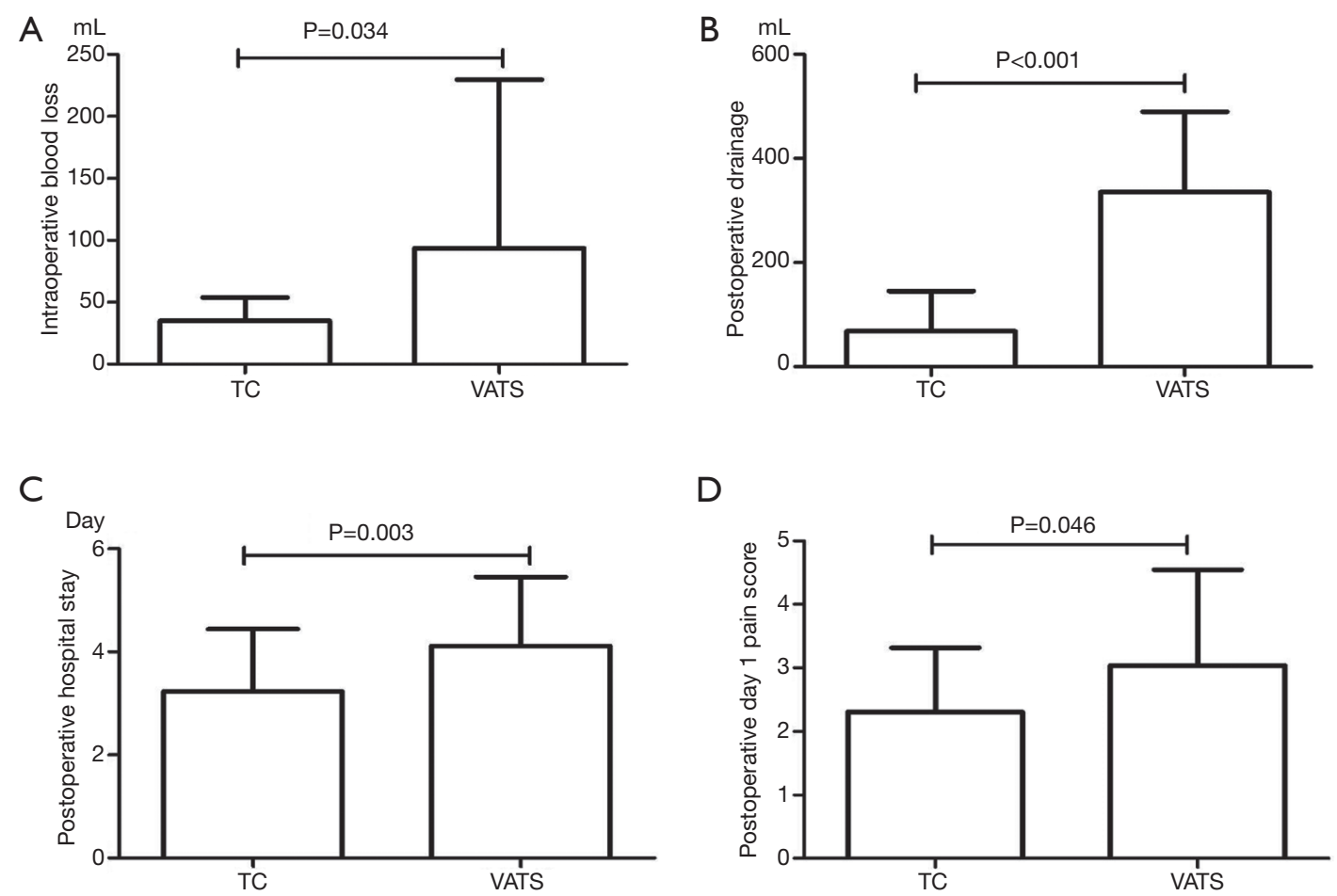

Figure 5 The comparison of perioperative outcomes between TC and VATS after propensity match. (A) Intraoperative blood loss was less in TC group. (B) Postoperative drainage was less in TC group. (C) Postoperative hospital stay was shorter in TC group. (D) Postoperative day 1 pain score was lower in TC group. TC, transcervical resection; VATS, video-assisted thoracoscopic surgery.

tumor sized between TC group and VATS group, the comparatively smaller size $(3.0 \mathrm{vs} .3 .3 \mathrm{~cm})$ in TC group might incur a selection bias due to the fact that TC is more likely to be used in the resection of smaller tumors. In order to minimize the potential confounding effect, a propensity score match (TC:VATS $=1: 1$ ) was further performed based on 4 variables (gender, age and tumor diameter and tumor characteristic). After propensity matching, TC group was associated with shorter incision length $(3.0 \pm 0.9$ vs. $5.1 \pm 1.1 \mathrm{~cm}$, $\mathrm{P}<0.001)$, less intraoperative blood loss $(35.1 \pm 18.7$ vs. $93.7 \pm 136.1 \mathrm{~mL}, \mathrm{P}=0.034)$, less postoperative drainage (65.6 \pm 76.8 vs. $335.0 \pm 154.9 \mathrm{~mL}, \mathrm{P}<0.001)$, lower pain score on postoperative day one $(2.3 \pm 1.0$ vs. $3.0 \pm 1.5, \mathrm{P}=0.046)$, shorter postoperative hospital stays $(3.2 \pm 1.2 v s .4 .1 \pm 1.3$ days, $\mathrm{P}=0.003)$ and less hospitalization expense $(22,252.3 \pm 4,761.7$ vs. 26,514.2 $\pm 4,052.8 \mathrm{CNY}, \mathrm{P}=0.002$ ) (Figure 5).

One patient in VATS group required conversion to thoracotomy due to intraoperative vessels damage and hemorrhage. Perioperative mortality was null in both groups. There was no complication in TC group, while one patient suffered from prolonged chest tube drainage due to the persistent air leak and two patients suffered from recurrent laryngeal nerve injury in VATS group.

\section{Discussion}

TC resection was firstly described by Schumacher and Roth in 1912 (15). Since then, TC has been suggested to be a successful and safe approach in numerous cases (16-19). In 1988, Cooper modified the sternal retractors that improved the visualization of the anterior mediastinum, and the short-term follow-up revealed that transcervical approach conferred equivalent outcomes compared with sternotomy $(21,22)$.

As mini-invasive an approach as VATS, TC represented an alternative approach in the treatment of mediastinal tumors. VATS has been demonstrated to be a safe procedure in the treatment of mediastinal tumors, and $\mathrm{Wu}$ et al. found uniport VATS was a safe and promising technique with short-term outcome not inferior to multiport VATS (13). However, whether TC was associated with better outcomes 
than VATS remained unclear. The study by Iacobone and colleagues including $51 \mathrm{TC}$ patients and 12 VATS patients found that there was no significant difference between 2 groups in hospital stay, operative time, operative pain and cosmetic results, but the authors suggested that TC might be still less invasive because the selective singlelung ventilation could be avoided (5). In addition, VATS was reported to cause postoperative chronic pain and chest wall numbness in some cases $(23,24)$. Deeb and colleagues suggested that TC is less invasive than VATS, particularly in terms of intercostal neuralgia (25).

To get a reliable result, we performed propensity score match between two groups. In our study, TC was less invasive with better perioperative outcomes compared with VATS, as indicated by the significant decrease in the incision length, Intraoperative blood loss, pain score on postoperative day one and postoperative stay. Moreover, there was no postoperative complication in TC group. No case in TC was converted to open surgery and required blood transfusion. However, in VATS group, two patients suffered from recurrent laryngeal nerve injury and one patient had persistent air leak, which could inevitably prolong their hospital stay. TC group was also associated with lower hospitalization costs. We thought two factors, the VATS-associated instruments and longer hospital stay, might pose the additional cost in the VATS group.

Despite the long history and excellent outcomes of TC, the indications for mediastinal tumors are still inconclusive. Iacobone reported that TC was preferred if tumor was in antero-superior locations (5). Callender found that only $25 \%$ of cases with tumors located more than $6 \mathrm{~cm}$ below clavicle were successfully performed by TC (26). Tumor in the deep location of mediastinum was considered as the main reason that TC failed. In addition, Deeb and colleagues reported that TC could be used when tumor size was less than $4 \mathrm{~cm}$ and the tumor did not invade surrounding structures in CT scan (25). However, several case reports showed that tumors more than $4 \mathrm{~cm}$ could also be accomplished by TC without any major complications and recurrence (27-29). Based on previous studies and our clinical experience, tumors invading surrounding structures and deep location tumors were considered as relative contraindication of TC resection, for it offered insufficient exposure when managing vascular and neural structures below the tumors. Therefore, we chose the antero-superior mediastinal tumors less than $5 \mathrm{~cm}$ in this study, and found that no postoperative complications occurred. We thought it was reliable to expand the diameter limitation to $5 \mathrm{~cm}$ for TC. TC was a safe and less invasive alternative for antero- superior mediastinal tumors less than $5 \mathrm{~cm}$, especially when VATS was not viable, such as the cases highly suspected of pleural adhesion.

This study has some limitations. First, it is a retrospective study, surgeon preference may affect the comparison of two groups and selection bias cannot be excluded. Although propensity score match may reduce the confounders. Further validation from prospective study is needed. Second, the pathological result is a heterogenous cohort, all the cases included are benign tumors or are in very early stage thymoma, they all have a very good prognosis. Therefore, we conclude that TC is less invasive for anterior-superior mediastinal tumors when comparing the perioperative outcomes, and these results have poor correlation with postoperative pathology. The role of TC for malignant mediastinal tumors could be evaluated in the further study.

\section{Conclusions}

TC is a safe and reliable technique for antero-superior mediastinal tumor, with better perioperative outcomes compared to VATS in terms of Intraoperative blood loss, postoperative drainage, pain score on postoperative day one and postoperative hospital stay.

\section{Acknowledgements}

None.

\section{Footnote}

Conflicts of Interest: The authors have no conflicts of interest to declare.

Ethical Statement: This study was approved by the Institutional Review Board of Tongji University (No. K18-112). Written informed consent was obtained from the patient for publication of this manuscript and any accompanying images.

\section{References}

1. Bacha EA, Chapelier AR, Macchiarini P, et al. Surgery for invasive primary mediastinal tumors. Ann Thorac Surg 1998;66:234-9.

2. Nakahara K. Current status of surgery for mediastinal tumors. Rinsho Kyobu Geka 1989;9:429-33.

3. Cansever L, Kocaturk CI, Cinar HU, et al. Benign posterior mediastinal neurogenic tumors: results of a 
comparative study into video-assisted thoracic surgery and thoracotomy (13 years' experience). Thorac Cardiovasc Surg 2010;58:473-5.

4. Nwariaku FE, Snyder WH, Burkey SH, et al. Inframanubrial parathyroid glands in patients with primary hyperparathyroidism: alternatives to sternotomy. World J Surg 2005;29:491-4.

5. Iacobone M, Mondi I, Viel G, et al. The results of surgery for mediastinal parathyroid tumors: a comparative study of 63 patients. Langenbecks Arch Surg 2010;395:947-53.

6. Davenport E, Malthaner RA. The role of surgery in the management of thymoma: a systematic review. Ann Thorac Surg 2008;86:673-84.

7. Zahid I, Sharif S, Routledge T, et al. Video-assisted thoracoscopic surgery or transsternal thymectomy in the treatment of myasthenia gravis? Interact Cardiovasc Thorac Surg 2011;12:40-6.

8. Russell CF, Edis AJ, Schalz DA, et al. Mediastinal parathyroid tumours: experience with 38 tumours requiring mediastinotomy for removal. Ann Surg 1981;193:805-9.

9. Sukumar MS, Komanapalli CB, Cohen JI. Minimally invasive management of the mediastinal parathyroid adenoma. Laryngoscope 2006;116:482-7.

10. Yim AP. Video-assisted thoracoscopic management of anterior mediastinal masses. Preliminary experience and results. Surg Endosc 1995;9:1184-8.

11. Yim AP, Kay RL, Ho JK. Video-assisted thoracoscopic thymectomy for myasthenia gravis. Chest 1995;108:1440-3.

12. Randone B, Costi R, Scatton O, et al. Thoracoscopic removal of mediastinal parathyroid glands: a critical appraisal of an emerging technique. Ann Surg 2010;251:717-21.

13. Wu CF, Gonzalez-Rivas D, Wen CT, et al. Comparative short-term clinical outcomes of mediastinum tumor excision performed by conventional VATS and single-port VATS. Medicine (Baltimore) 2015;94:e1975.

14. Cupisti K, Dotzenrath C, Simon D, et al. Therapy of suspected intrathoracic parathyroid adenomas.

Experiences using open transthoracic approach and videoassisted thoracoscopic surgery. Langenbecks Arch Surg 2002;386:488-93.

15. Schumacher ED, Roth J. Thymektomie bei cenem fall von morbus basedowii mit myasthenie. Med Chir 1912;25:746.

16. Kirschner PA, Osserman KE, Kark AE. Studies in myasthenia gravis. Transcervical total thymectomy. JAMA 1969;209:906-10.

17. Daddi N, Nakajima T, Tagawa T, et al. Transcervical resection of an ectopic mediastinal parathyroid adenoma. Ann Thorac Surg 2012; 94:1740.

18. Ducic Y, Oxford L. Transcervical elective superior mediastinal dissection for thyroid carcinoma. Am J Otolaryngol 2009;30:221-4.

19. Sakuraba M, Miyasaka Y, Kodu Y, et al. The Cervical anterior approach for the resection of superior posterior neurogenic tumor: a case report. Ann Thorac Cardiovasc Surg 2012;18:42-4.

20. Farrar JT, Young JP Jr, LaMoreaux L, et al. Clinical importance of changes in chronic pain intensity measured on an 11-point numerical pain rating scale. Pain 2001;94:149-58.

21. Cooper JD, Al-Jilaihawa AN, Pearson FG, et al. An improved technique to facilitate transcervical thymectomy for myasthenia gravis. Ann Thorac Surg 1988;45:242-7.

22. Meyers BF, Cooper JD. Transcervical thymectomy for myasthenia gravis. Chest Surg Clin N Am 2001;11:363-8.

23. Searle RD, Simpson MP, Simpson KH, et al. Can chronic neuropathic pain following thoracic surgery be predicted during the postoperative period? Interact Cardiovasc Thorac Surg 2009;9:999-1002.

24. Miyazaki T, Sakai T, Tsuchiya T, et al. Assessment and follow-up of intercostal nerve damage after video-assisted thoracic surgery. Eur J Cardiothorac Surg 2011;39:1033-9.

25. Deeb ME, Brinster CJ, Kucharzuk J, et al. Expanded indications for transcervical thymectomy in the management of anterior mediastinal masses. Ann Thorac Surg 2001;72:208-11.

26. Callender GG, Grubbs EG, Vu T, et al. The fallen one: the inferior parathyroid gland that descends into the mediastinum. J Am Coll Surg 2009;208:887-93

27. Haldar A, Thapar A, Khan S, et al. Day-case minimally invasive excision of a giant mediastinal parathyroid adenoma. Ann R Coll Surg Engl 2014;96:e21-3.

28. Power C, Kavanagh D, Hill AD, et al. Unusual presentation of a giant parathyroid adenoma: report of a case. Surg Today 2005;35:235-7.

29. Netterville JL, Coleman SC, Smith JC, et al. Management of substernal goiter. Laryngoscope 1998;108:1611-7.

Cite this article as: Zhu XS, Song N, Song NC, Sihoe AD, He WX, Liu M, Jiang GN, Zhang P. Comparison of the perioperative outcomes in antero-superior mediastinal tumor resection performed by transcervical resection and videoassisted thoracoscopic surgery. J Thorac Dis 2018;10(12):68386845. doi: $10.21037 /$ jtd.2018.11.114 